\title{
ESTUDO DA ESTABILIDADE DO BIOSSURFACTANTE PRODUZIDO POR PSEUDOMONAS CEPACIA
}

\author{
R. C. F. SOARES DA SILVA ${ }^{1}$, D. G. ALMEIDA ${ }^{1}$, P. P. F. BRASILEIRO ${ }^{2}$, B. G. FREITAS ${ }^{2}$, R. D. \\ RUFINO $^{2}$; J. M. LUNA ${ }^{2}$ e L. A. SARUBBO ${ }^{1,2}$ \\ ${ }^{1}$ Universidade Federal Rural de Pernambuco, Rede Nordeste de Biotecnologia - RENORBIO; \\ FACEPE, Centro de Gestão de tecnologia e Inovação - CGTI. \\ ${ }^{2}$ Universidade Católica de Pernambuco e Centro de Ciência e Tecnologia; CAPES, FACEPE, \\ UNICAP, TERMOPE, CNPq, ANEEL. \\ E-mail para contato: ritaf78@gmail.com
}

\begin{abstract}
RESUMO - Os inovadores biossurfactantes surgiram para substituir os surfactantes químicos, em virtude das propriedades tensoativas, maior estabilidade e biodegradabilidade. Nesse sentido, o presente trabalho investigou a estabilidade do biossurfactante produzido por Pseudomonas cepacia CCT6659 em meio contendo $2 \%$ de óleo de canola residual, $3 \%$ de milhocina e $0,2 \%$ de NaNo3, durante 60 horas, e agitação de 250rpm. No líquido metabólico livre de células foi adicionado $0,2 \%$ do sal sorbato de potássio para a conservação de suas propriedades surfactantes. As condições de aquecimento, $\mathrm{pH}$ e salinidade foram aplicadas para perceber os melhores resultados de aplicação do biotensoativo durante 120 dias. Os resultados mais expressivos das atividades do biossurfactante produzido foram tensão superficial de $25,92 \mathrm{mN} / \mathrm{m}$, capacidade emulsificante de $99 \%$ para o óleo motor e dispersante em água do mar de $53,5 \%$. Portanto, foi comprovado o potencial de possíveis aplicações desse agente surfactante em processos de descontaminação ambiental.
\end{abstract}

\section{INTRODUÇÃO}

Os tensioativos são compostos químicos que reduzem a tensão superficial de um líquido. Tais compostos têm uma predileção para interfaces de polaridades diferentes (liquido-ar ou líquido-líquido) e são solúveis em ambos os solventes orgânicos (não polar) e aquosa (polares). Estas propriedades são originários de estruturas anfifílicas (ou anfipáticas), que compreendem tanto hidrofílica (cabeça) e grupos hidrofóbicos (cauda) (Luna et al., 2013).

Biossurfactantes são surfactantes sintetizados como produtos metabólicos de diferentes microorganismos. A indústria de petróleo constitui o maior mercado para os biossurfactantes, onde podem ser usados para biorremediação/dispersão de manchas oleosas, tanto no solo quanto no mar, na remoção/mobilização de óleos incrustados em tanques de estocagem em rochas e areia do mar, aumentando a sua recuperação e nas áreas de proteção ao meio ambiente (Rufino et al., 2008). 
Atualmente, a maioria dos compostos de superfície-ativa no mercado são principalmente de origem sintética. O principal fator que restringe o uso de biossurfactantes no mercado é seu custo de produção quando comparado com seus similares sintéticos. $\mathrm{O}$ uso alternativo de substratos de baixo custo, tais como resíduos agroindustriais, é uma importante estratégia para reduzir os custos associados ao processo e proporcionar o desenvolvimento industrial da produção de biossurfactantes (Costa, 2010).

As bactérias, juntamente com as arqueobactérias, são os maiores responsáveis pela produção destes compostos tensoativos; bactérias das famílias Pseudomonacea e Bacillacea são capazes de produzirem biossurfactantes eficientes na remoção de petróleo e seus derivados poluentes de água (Bueno, 2010). Raminolipídios produzidos por Pseudomonas são os melhores agentes tensoativos glicolipídicos conhecidos e a sua gama de aplicações potenciais nas indústrias de cerâmica, alimentos, cosméticos, produtos farmacêuticos, metal, papel, e nas para aplicações ambientais, tal como a biorremediação (Silva et al., 2013).

Dessa forma, as questões ambientais e econômicas motivaram a realização deste estudo, que apresenta a produção de biossurfactante por uma cepa de Pseudomonas cepacia, CCT6659, usando um meio mineral previamente otimizado de baixo custo suplementado com óleo de canola (residual de fritura) e milhocina como substratos (Silva et al., 2013).

Contudo, os inovadores biossurfactantes substituem os dispersantes químicos devido suas inúmeras aplicações e pelos benefícios ambientais avaliados em relação a biodegradabilidade e baixa toxicidade. A estabilidade é um fator essencial para viabilizar a produção em larga escala, principalmente, de um produto biotecnológico que demora a ser produzido perante a urgência da aplicação em um desastre petrolífero. Todo o óleo precisa ser retirado do oceano em cerca de 24 horas após o derramamento. Logo, a durabilidade precisa ser elevada com a finalidade de utilização imediata (Marchant; Banat, 2012).

Diante do exposto, o presente estudo teve como objetivo estudar o comportamento do biossurfactante produzido por Pseudomonas cepacia, CCT6659, sob diversas propriedades apresentadas e sob condições específicas, no decorrer do tempo e na manutenção do biossurfactante por adição de um sal químico como aditivo.

\section{MATERIAL E METODOS}

\subsection{Micro-organismos}

Para a produção dos biossurfactantes microbianos foi utilizada a bactéria Pseudomonas cepacia (CCT6659). A cultura foi mantida em meio AN (Ágar Nutriente), à temperatura de $5^{\circ} \mathrm{C}$.

\subsection{Inóculo}

Para o crescimento do micro-organismo foi utilizado o meio $\mathrm{CN}$ (Caldo Nutriente) composto por 3,0g extrato de carne; 5,0g peptona; 5,0g NaCl; água destilada q.s.p. $1000 \mathrm{ml}$; $\mathrm{pH}$ 
7,0; temperatura de $28^{\circ} \mathrm{C}$ e $150 \mathrm{rpm}$, por 16 horas até obtenção de uma D.O (Densidade Óptica) de 0,7 (correspondente a um inóculo de $10^{7}$ U.F.C. $/ \mathrm{mL}$ ) a $600 \mathrm{~nm}$ com a concentração de $1 \%$ $(\mathrm{v} / \mathrm{v})$.

\subsection{Meio de produção}

O meio mineral descrito por Dubey e Juwarkar (2001), foi suplementado com $2 \mathrm{ml}$ de óleo de canola (residual de fritura), $3 \mathrm{~mL}$ de milhocina como fonte de carbono e $0,2 \mathrm{~g} / \mathrm{L}$ de NaNo3 para a produção do biossurfactante. Após o preparo dos meios, o pH foi ajustado para 7,0 e os meios foram autoclavados a $121^{\circ} \mathrm{C}$ por 20 minutos.

\subsection{Produção do biossurfactante}

As fermentações para produção do biossurfactante foram realizadas em frascos de erlenmeyer de $500 \mathrm{ml}$ de capacidade, contendo $100 \mathrm{ml}$ do meio de produção, e incubados com $1,5 \%$ do pré-inóculo. Os frascos foram mantidos sob agitação orbital de $250 \mathrm{rpm}$ durante 60 horas, à temperatura de $28^{\circ} \mathrm{C}$. Ao fim do cultivo, as amostras foram centrifugadas e filtradas. $\mathrm{O}$ biossurfactante bruto serão utilizados para avaliação das propriedades surfactantes.

\subsection{Estabilização do biotensoativo e determinação das propriedades surfactantes}

A estabilização do surfactante microbiano ocorreu após a adição de $0,2 \%$ do sal sorbato de potássio e o líquido dividido em vidros de armazenamento, em triplicata, para a verificação das propriedades surfactantes nos períodos de 0, 15, 30, 45, 90 e 120 dias. Em cada um desses dias, foram alteradas as condições de potencial hidrogeniônico para 5, 7 e 9; adicionadas percentagens de salinidade 1,3 e $5 \%$ do volume a ser estudado e aquecidas as amostras durante $30 \mathrm{~min}$ a 40 e $50{ }^{\circ} \mathrm{C}$. Para avaliar as propriedades surfactantes, foram mensuradas as tensões superficiais e os índices de emulsificação e dispersão.

As tensões foram medidas pelo método do anel DU NUOY no tensiômetro KSV Sigma 70 (Finland). A superficial foi mensurada pela força limite $(\mathrm{mN} / \mathrm{m})$ para suspender o anel de platina no líquido metabólico livre de células até a interface ar-líquido e a interfacial entre o biossurfactante e o hidrocarboneto n-hexa-decano.

Para a determinação das emulsões, foram colocados em tubos de ensaio, $2 \mathrm{ml}$ do biossurfactante acoplados isovolumetricamente a três compostos apolares: óleos motor, de milho e de soja a $25{ }^{\circ} \mathrm{C}$ e $1 \mathrm{~atm}$. Esses recipientes foram agitados em vórtex durante 2 minutos e deixados, durante 24 horas, em repouso. $\mathrm{O}$ índice de emulsificação foi calculado pela razão entre a altura da emulsão e a altura total da mistura, sendo o valor multiplicado por 100 (Cooper; Goldenberg, 1987). 


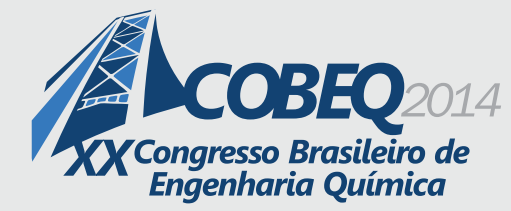

A capacidade de dispersão e agregação do óleo motor foi simulada em placa de Petri (15 $\mathrm{cm}$ de diâmetro), contendo $100 \mathrm{ml}$ da água do mar, coletada nas proximidades do sistema de captação do gerador de energia da Termelétrica Pernambuco. Uma quantidade desse óleo foi colocada no centro da placa, adicionando posteriormente diferente proporções do surfactante microbiano: 1:2, 1:8 e 1:25 (v/v) (biossurfactante/óleo motor). Os valores foram medidos pela percentagem do diâmetro do halo, formado com a adição do biossurfactante, pelo da placa, e o teste de agregação foi apenas visual (Morikawa et al, 2000).

\section{RESULTADOS E DISCUSSÃO}

A tensão superficial é a força de atração existente entre as moléculas dos líquidos. A redução das tensões superficial e interfacial é considerada como principal parâmetro para detecção de um composto tensoativo em um determinado meio (Rufino et al., 2008; Luna et al., 2013). A figura 1 mostra os valores de tensão superficial do líquido metabólico livre de células produzido por Pseudomonas cepacia (CCT6659) após submetido ao processo de conservação por adição de sal sorbato de potássio, e variações de $\mathrm{pH}(5,7$ e 9$)$, temperatura $\left(40,50^{\circ} \mathrm{C}\right)$ e adição de diferentes concentrações de $\mathrm{NaCl}(1,3$ e $5 \%)$ para determinação de sua estabilidade frente a condições ambientais extremas.

Segundo (Souza et al., 2014) uma propriedade de grande importância para aplicações industriais e biotecnológicas é a estabilidade da maioria dos biossurfactantes em diferentes condições, suportando altas temperaturas de autoclavagem $\left(121^{\circ} \mathrm{C}\right.$ por $\left.20 \mathrm{~min}\right)$ e também as baixas temperaturas ( $-18 \mathrm{C}^{\circ}$ durante 6 meses), e pH (5 e11 ) encontrado no ambiente. As tensões superficiais do líquido metabólico em questão submetido a variações de $\mathrm{pH}$ apresentaram as seguintes alterações: durante os 120 dias de experimento as melhores condições obtidas para a tensão do líquido metabólico submetido ao $\mathrm{pH} 7$ permaneceu em torno de $25,92 \mathrm{mN} / \mathrm{m}$, salinidade de $3 \%$ com $26,63 \mathrm{mN} / \mathrm{m}$ e aquecimento a $50{ }^{\circ} \mathrm{C}$ com $26,66 \mathrm{mN} / \mathrm{m}$. Com esses resultados para o método de conservação foi evidenciado que o biossurfactante apresentou ótima estabilidade frente as várias condições adversas no decorrer do período de 120 dias. Conforme mostra a Figura 1.

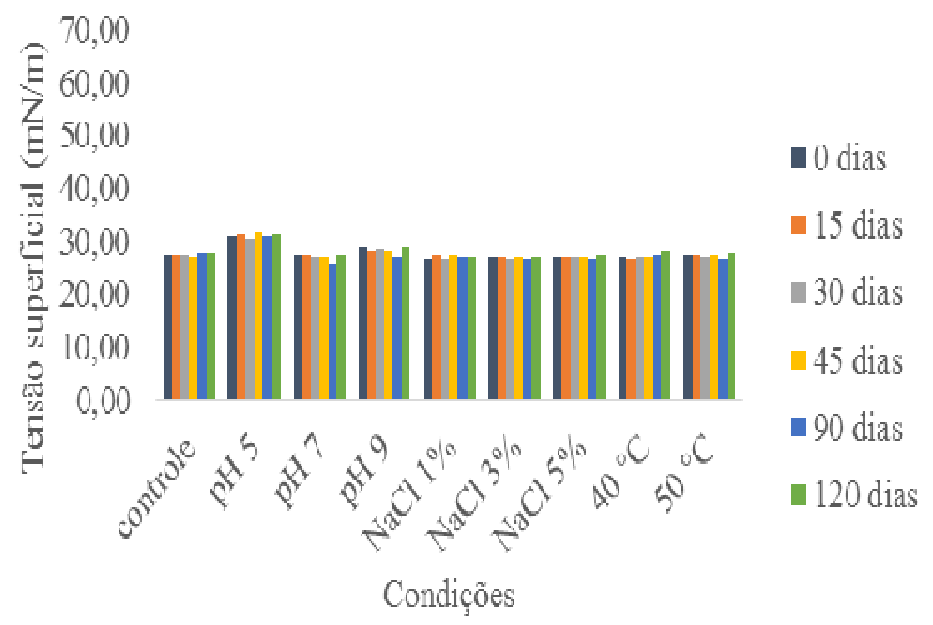




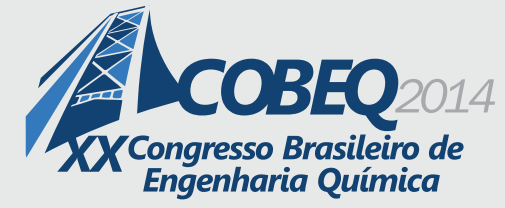

Figura 1 - Tensões superficiais do biossurfactante adicionado de sorbato de potássio a $0,2 \%$, durante 120 dias, sob variação de $\mathrm{pH}, \mathrm{NaCl}$, aquecimento e com o controle das condições

Os índices de emulsificação foram avaliados em três tipos de óleos: Motor (A), Milho (B), e Soja (C) conforme a Figura 2. O primeiro óleo apresentaram os maiores índices de emulsificação com quatro porcentagens em $99 \%$ até 90 dias de experimento. Para as três faixas de $\mathrm{pH}$ as melhores condições foram nos três tipos de óleo, no aquecimento as temperaturas de $40^{\circ}$ e $50^{\circ} \mathrm{C}$, e adição de salinidade de $3 \%$. Nos índices de emulsificação em óleo motor, foram percebidas consideráveis elevações em todas as percentagens diante do controle do biossurfactante. Esse acontecimento foi de encontro ao ocorrido nas figuras 2-A, com óleo de motor, e 2-B, com óleo de milho.

A

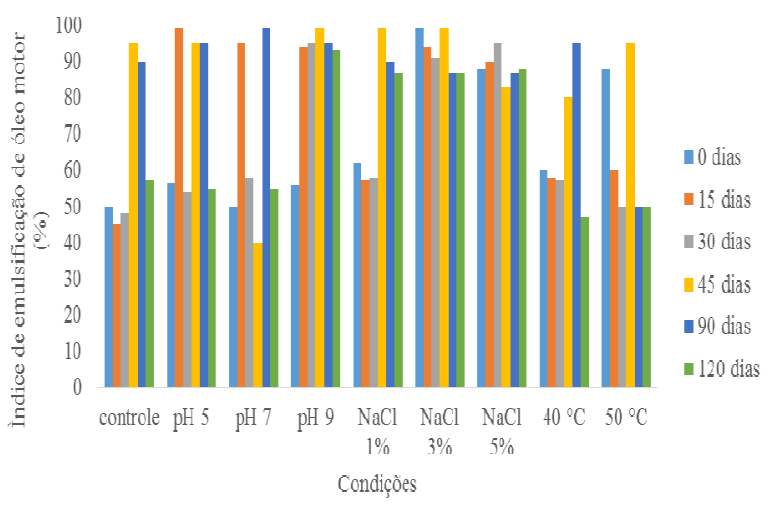

B

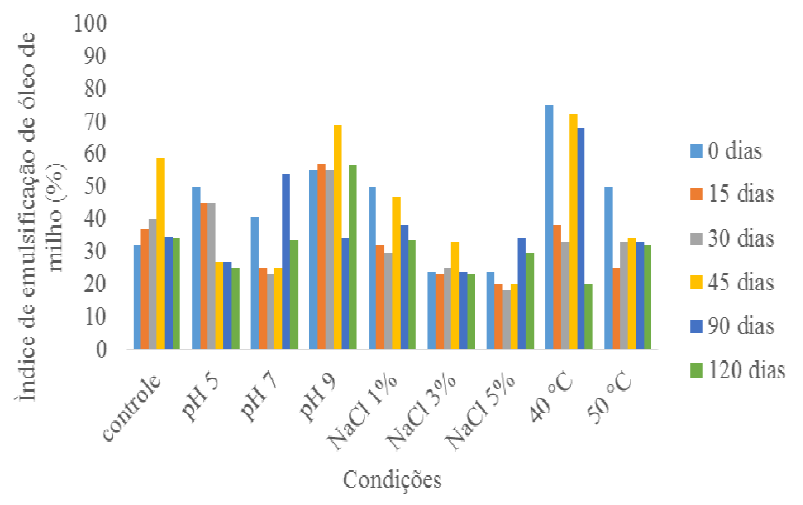

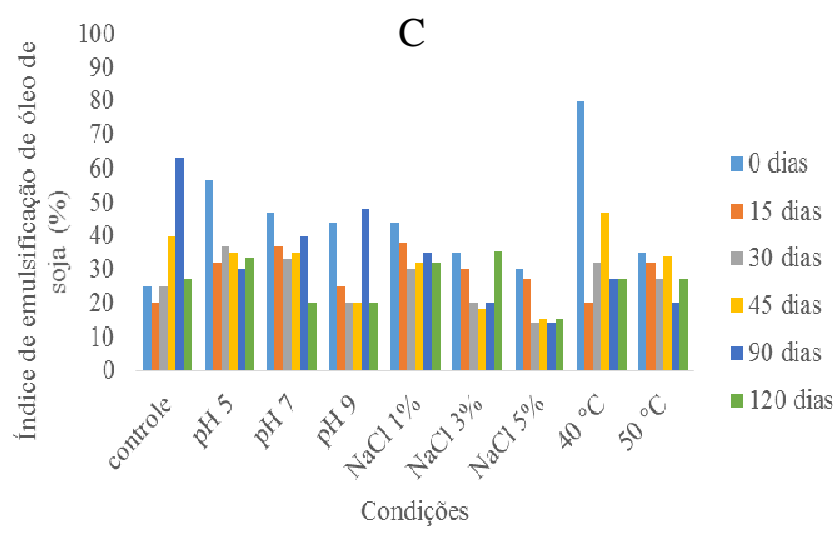

Figura 2 - Índices de emulsificação do biossurfactante adicionada de sorbato a 0,2\%, durante 120 dias, sob variação de $\mathrm{pH}, \mathrm{NaCl}$ e aquecimento nos óleos: (A) motor, (B) milho e (C) soja. 
Nos índices das dispersões, os percentuais foram mais elevados frente às alterações de $\mathrm{pH}$, com um valor máximo de 53,5\%, aos 120 dias com pH 9 e nas proporções 1:2 e 1:8 (v/v) de biossurfactante para óleo motor. As melhores condições foram observadas nas dispersões 1:2 e 1:8 (v/v), $\mathrm{pH} 9$, a salinidade $3 \%$ e aquecimento a $50^{\circ} \mathrm{C}$. A dispersão $1: 25(\mathrm{v} / \mathrm{v})$ não diferiu consideravelmente em relação a proporção 1:8, conforme a Figura 3.

A

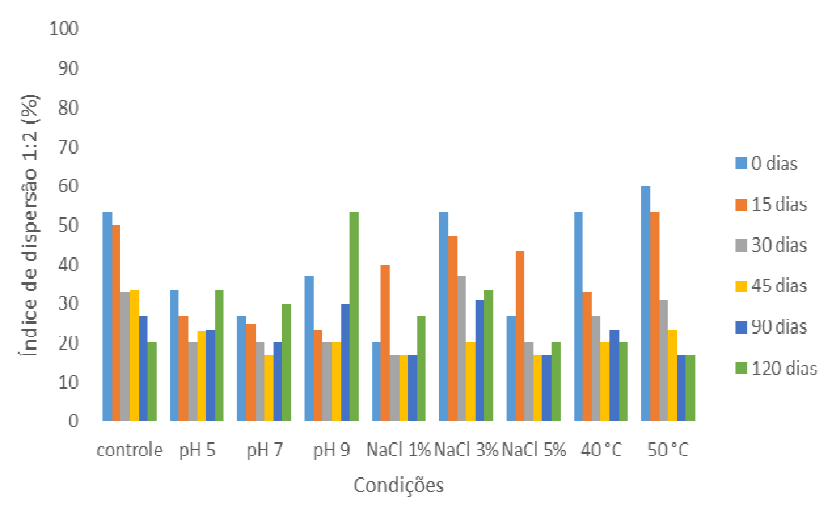

B

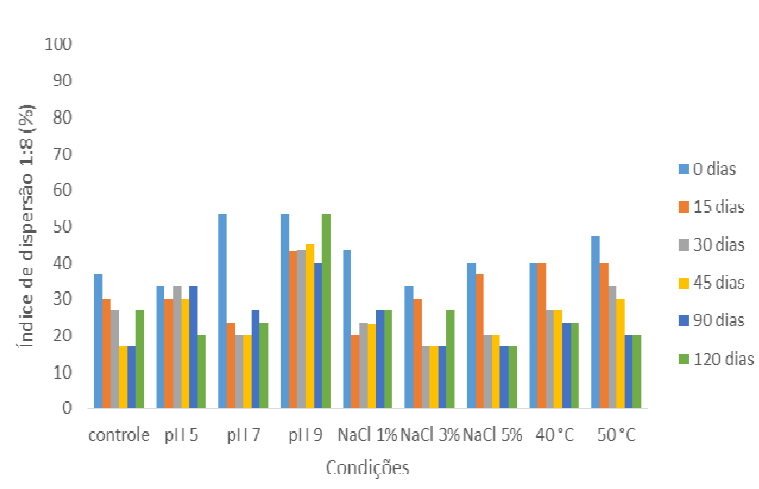

$\mathrm{C}$

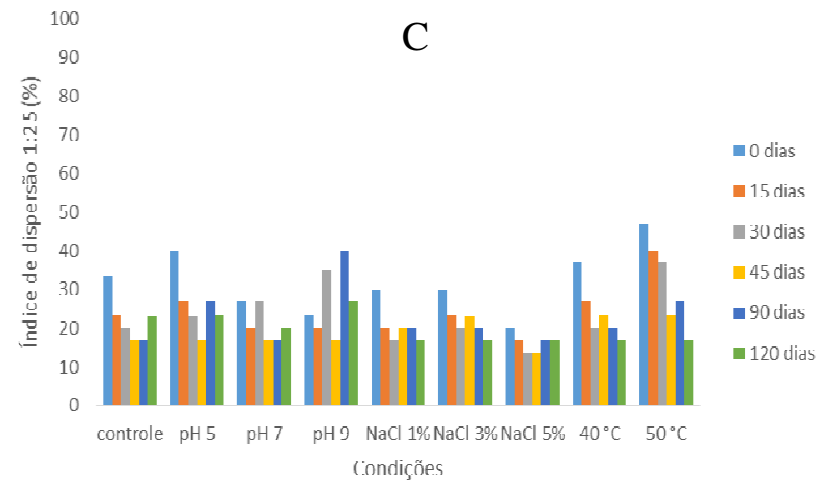

Figura 3 - Índices de dispersões do biossurfactante adicionado de sorbato a 0,2\%, durante 120 dias, sob variação de $\mathrm{pH}, \mathrm{NaCl}$ e aquecimento, nas proporções de biossurfactante/óleo (v/v): (A) 1:2, (B) $1: 8$ e (C) $1: 25$ 


\section{CONCLUSÕES}

1. As tensões superficiais se mantiveram estáveis, não apresentando alterações significantes, tornando o processo de conservação muito eficiente;

2. As emulsificações obtiveram os melhores resultados no óleo motor, principalmente com a alteração do pH 5 e 9 e com o aquecimento de $40{ }^{\circ} \mathrm{C}$ e $3 \%$ de salinidade;

3. Os índices de dispersão foram elevados nas três proporções estudadas principalmente nas proporções 1:2 e 1:8. Às melhores condições foram os potenciais de hidrogênio 7 ou 9, preferindo-se um $\mathrm{pH} 9$ por estar próximo ao do mar; a salinidade de 1 ou $3 \%$; ou o aquecimento a $50{ }^{\circ} \mathrm{C}$;

4. O biossurfactante de Pseudomonas cepacia CCT6659, apresentou resultados bastante satisfatórios quanto ao método de formulação testado pelo processo de adição de $0,2 \%$ de sorbato de potássio. Podendo ser utilizado como coadjuvante na biorremediação de ambientes aquáticos impactados por contaminação de derivados de petróleo.

\section{REFERÊNCIAS}

BUENO, S. M.; SILVA, A. N. E GARCIA-CRUZ, C. H. Estudo da produção de biossurfactante em caldo de fermentação. Quim. Nova, v. 33, n. 7, p.1572-1577, 2010.

COSTA, S. G. V. A. O.; NITSCHKE, M.; LÉPINE, L.; DÉZIEL, E. E CONTIERO, J. Structure, properties and applications of rhamnolipids produced by Pseudomonas aeruginosa L2-1 from cassava wastewater. Elsevier, v.45, p.1511-1516, 2010.

COOPER, D. G.; GOLDENBERG, B. G. Surface-Active Agents from two Bacillus Species. Applied and Enviromental Microbiology, v. 53, p. 224-229, 1987.

LUNA, J. M.; RUFINO, R. D.; SARUBBO, L. A.; CAMPOS-TAKAKI, G. M. Characterization, surface properties and biological activity of a biosurfactant produced from industrial waste by Candida sphaerica UCP0995 for application in the petroleum industry. Coll. Surf. B: Biointer., v. 102, p. 202-209, 2013.

DUBEY, K, JUWAKAR, A. Distillery and curd whey wastes as viable alternative sources for biosurfactant production. World Journal Microbiology and Biotechnology, v. 17, p. 61- 69. 2001.

MARCHANT, R.; BANAT I. M. Microbial biosurfactants: challenges and opportunities for future exploitation. Trends Biotechnol.; v.11, p. 558-565, 2012.

MORIKAWA, M et al. A study on the structure-function relationship of lipopeptide biosurfactants. Biochimica et Biophysica Acta, v. 1488, p. 211-218, 2000. 
RUFINO, R. D.; SARUBBO, L.A. E CAMPOS-TAKAKI, G.M. Enhancement of stability of biosurfactant produced by Candida lipolytica using industrial residue as substrate. World Journal of Microbiology and Biotechnology, v.23, p.729-734, 2008.

SILVA, R.C.F.S. RUFINO, R.D., LUNA, J.M., FARIAS, C.B.B., SANTOS, V.A., FILHO, H.J.B.L., SARUBBO, L.A. Enhancing biosurfactant production from Pseudomonas cepacia CCT6659 by optimizing nutritional parameters using a response surface methodology. Tenside Surf. Det., v.50, n.2, p. 137-142, 2013.

SOUZA, E. C.; THEREZA C. VESSONI-PENNA; OLIVEIRA, R. P. S. Biosurfactant-enhanced hydrocarbon bioremediation: An overview. International Biodeterioration \& Biodegradation. v.89 p. 88 e $94,2014$. 